\title{
Computational Smart Polymer Design based on Elastin Protein Mutability
}

\author{
Anna Tarakanova ${ }^{1}$, Wenwen Huang ${ }^{2}$, Anthony S. Weiss ${ }^{3}$, David L. Kaplan ${ }^{2}$, Markus J. Buehler ${ }^{\text {* }}$ \\ ${ }^{1}$ Laboratory for Atomistic and Molecular Mechanics, Department of Civil and Environmental \\ Engineering, Massachusetts Institute of Technology, Cambridge, MA, USA \\ ${ }^{2}$ Department of Biomedical Engineering, Tufts University, Medford, MA, USA \\ ${ }^{3}$ School of Life and Environmental Sciences and Charles Perkins Centre, The University of \\ Sydney, Sydney, NSW, Australia. \\ *Corresponding author, $\underline{\text { mbuehler@MIT.EDU }}$
}

\begin{abstract}
Soluble elastin-like peptides (ELPs) can be engineered into a range of physical forms, from hydrogels and scaffolds to fibers and artificial tissues, finding numerous applications in medicine and engineering as "smart polymers". Elastin-like peptides are attractive candidates as a platform for novel biomaterial design because they exhibit a highly tunable response spectrum, with reversible phase transition capabilities. Here, we report the design of the first virtual library of elastin-like protein models using methods for enhanced sampling to study the effect of peptide chemistry, chain length, and salt concentration on the structural transitions of ELPs, exposing associated molecular mechanisms. We describe the behavior of the local molecular structure under increasing temperatures and the effect of peptide interactions with nearest hydration shell water molecules on peptide mobility and propensity to exhibit structural transitions. Shifts in the magnitude of structural transitions at the single-molecule scale are explained from the perspective of peptide-ion-water interactions in a library of four unique elastin-like peptide systems. Predictions of structural transitions are subsequently validated in experiment. This library is a valuable resource for recombinant protein design and synthesis as it elucidates mechanisms at the single-molecule level, paving a feedback path between simulation and experiment for smart material designs, with applications in biomedicine and diagnostic devices.
\end{abstract}

Key Words: Elastin-like peptide (ELP), inverse temperature transition, peptide-water interaction, single-molecule, protein simulation library

Submitted to: Biomaterials, Manuscript number No. jbmt38279 (revised)

\section{Introduction}

Elastin is an extracellular matrix protein that imparts reversible elastic recoil and resilience to extensible tissues, including the lung, blood vessels, elastic ligaments and skin. Beyond its impressive mechanical elasticity, elastin and artificial elastin-like peptides (ELPs) have been demonstrated, through transmission electron microscopy, circular dichroism and nuclear magnetic resonance [1-5], to exhibit an inverse temperature transition, a reversible propensity to aggregate upon increasing temperature [2, 6-12]. Above a specific temperature, highly dependent on the chemistry and environment of the ELP system, the formation of an aggregating coacervate phase takes place. This is similar to the natural 
coacervation process of tropoelastin, the precursor molecule to elastin, in the process of elastic fiber formation [13].

The stimuli-responsive quality confers controllable functionalities to ELPs, and in conjunction with their biocompatibility and biodegradability, makes them effective candidates as a platform for biomedical materials. ELPs have been designed into a range of material forms, including nanoparticle self-assemblies [24, 25] and hydrogels [1, 26, 27]. The tunability of the ELP phase transitions has been used in biomedical applications from drug delivery [28-30] to tissue engineering [31]. Examples of such applications include elastin-based nanoparticles that have been designed to display cell-penetrating peptide motifs for controlled uptake into cells; thermal targeting of tumors by creation of a diffusion gradient of a drug into the tumor; tumor targeting with local hyperthermia; ELP conjugates for cancer therapy; and sustained release delivery systems for controlled release of peptide drugs to treat type 2 diabetes [32-36].

Underlying the application of ELPs in bioengineering and biomedicine is a need to understand fundamental design principles and molecular mechanisms yielding the stimuliresponsive quality to ELPs. In the last half century, a series of pivotal studies have contributed to the understanding of transition effects in ELPs. Urry's laboratory conducted extensive studies to understand the effects of sequence modification on transition temperature by varying guest residue X in VPGXG repeat sequences, coining the "inverse temperature transition" term [14]. They found a correlation between hydrophobicity index of guest residues and the transition temperature $\mathrm{T}_{\mathrm{t}}$, such that hydrophobic residues reduced $\mathrm{T}_{\mathrm{t}}$, while polar and hydrophilic residues increased $\mathrm{T}_{\mathrm{t}}$ [37].

Structural transitions in elastin have also been discussed in the context of elastin's elasticity by Tamburro and others, using the reductionist approach to study short synthesized glycine-rich peptides representative of elastin. In this description, a shifting set of sliding $\beta$-turns, polyproline II and unordered structures directs conformational flexibility in the protein, establishing a general equilibrium between folded and extended structures $[38,39]$. Tamburro's team studied the effect of sequence, chain length and environmental conditions on elastin's propensity to coacervate, suggesting particular functional roles for different domains based on these findings [39-41].

A number of experimental studies from a biomaterials design perspective have considered the possibility of developing polymeric ELP materials with introduced temperaturemodulated switches. Stimuli include sequence chemistry modification [16-19], changes in salt concentration [17], molecular weight [19-21], pH [20, 22], and light [23]. Beyond this, Chilkoti's team created a series of quantitative models for making specific predictions based on a set of sequence and solution parameters [11, 22, 42], with an emphasis on applications for drug delivery system development $[43,44]$. Several experimental groups have also considered the particular role of water and hydration on ELP transitions $[45,46]$ and related elasticity [47].

Supplementing experimental work, computational groups have developed ELP models. Several teams have used classical molecular dynamics to explore the molecular basis of elasticity and inverse temperature transition in ELPs [48-50]. The question of structure in ELPs has inspired studies that showed the sequence-dependent propensity of ELPs to 
assume amyloidogenic qualities [51,52]. Yet other studies have instead focused on ELP modeling to understand peptide receptor interactions and molecular implications in biological activity [53, 54].

The present work builds on existing literature to expand the understanding of fundamentals of ELP transitions and factors that influence this behavior. Unlike earlier computational work where ELPs systems have been studied primarily using classical molecular dynamics for a sparsely distributed temperature range, this study introduces Replica Exchange Molecular Dynamics (REMD) to improve sampling and identify specific transition regions not previously captured in experiment nor simulation by considering 60 densely-distributed temperature systems. Using this method, we examine how varied conditions and triggers influence mechanisms involved in the presence and absence of transitions for different ELP sequences at the single-molecule scale.

We focus on intramolecular phenomena in order to understand basic events at the singlemolecule and peptide domain level. A fundamental understanding at the sub-molecular scale can provide a foundation to extend this work for studying intermolecular events. The pentapeptide repeat unit VPGVG, and its permutation, GVGVP, are considered representative ELP polymer building blocks. Here we choose the GVGVP peptapeptide as proposed by Urry and used since by others as a model ELP $[9,55,56]$. Our work considers molecular compactness and secondary structure, hydrogen bonding organization, and distribution and arrangement of water in modulating ELP behavior based on changes in chemistry, salt concentration, and peptide chain length.

The approach introduced here can be extended to studying other synthetic or protein polymers for precise phase transition characterization at the molecular scale. For example, silk and silk-inspired sequences have shown a propensity to undergo temperature-induced phase transitions $[57,58]$ and are popular candidate materials for biomedical applications [59]. A number of synthetic polymers also show temperature-dependent phase transition functionality [60] which can be further tuned for precise behavior and control with a modeling-experimental approach described in this work.

Based on our findings, we propose modeling as a complementary tool to provide fast, reliable predictions based on core molecular mechanisms, because current methods are inadequate and do not consider the contributions of fast and slow molecular dynamics on the one hand; and the balancing of interactions between peptide, ions and water on the other. Furthermore, understanding single molecule behavior mechanisms is a necessary prelude to studying intermolecular events such as coacervation. This approach can be used to quickly screen the sequence space from a library of candidate sequences, in order to define sequences that might be useful experimentally (Figure 1).

\section{Methods}

\section{Molecular Simulation Setup}

Input structures for simulation are created based on elastin-like protein polymer (ELP) sequences of the form [(GVGVP)(GXGVP)(GVGVP) $]_{n}$ in single amino acid letter code (SI 
Figure 1a). $X$ is an interchangeable amino acid considered for shifting and modifying transition temperatures and $n$ (equal to 1 or 2 ) determines sequence length. The systems considered in this study are described in SI Table 1. X-residue identity, chain length, and $\mathrm{NaCl}$ concentration are considered.

Extended conformations of the sequences are built using CHARMM version 24b1 [61]. The CHARMM force field is widely used for studying proteins [62]. It has been used to study other fibrous proteins, such as collagen $[63,64]$, and has been shown to effectively capture the inverse temperature transition of elastin [65]. Following sequence construction, all subsequent system modifications and simulations are carried out using GROMACS version 5.01 [66]. Each system includes the protein sequence [(GVGVP)(GXGVP)(GVGVP)]n in a rectangular water box in $1 \mathrm{M}$ or $100 \mathrm{mM} \mathrm{NaCl}$, with periodic boundary conditions (SI Figure 1b). The number of atoms in each system studied here is indicated in SI Table $\mathbf{1 .}$ The CHARMM27 force field is used, which includes CHARMM22 and CMAP for proteins [62].

First, the structure is relaxed to ensure no steric clashes using energy minimization through the steepest descent algorithm. Then, solvent and ions are equilibrated around the protein, where the protein is fixed, through two equilibration stages, each 100 ps in length. The first phase is equilibration in an NVT ensemble to stabilize temperature, followed by a second stage in an NPT ensemble to stabilize system pressure. After the solvent is equilibrated, the protein restraint is removed and the protein and solvent are equilibrated in an NPT ensemble for an additional 100 ps. After this stage, final structures are input into Replica Exchange Molecular Dynamics (REMD) simulations [67] (SI Figure 1c). The Berendsen thermostat [68] is used for temperature coupling and the Parrinello-Rahman barostat [69] is used for pressure coupling. The short range electrostatic interactions and Lennard-Jones interactions are evaluated with a cutoff of $10 \AA$ A. Particle-mesh Ewald summation [70] is used to calculate long range electrostatic interactions with a grid spacing of $1.6 \AA$ and a fourth order interpolation.

\section{Replica Exchange Molecular Dynamics}

We build on existing work by using accelerated molecular modeling methods, specifically Replica Exchange Molecular Dynamics (REMD), to introduce better sampling of the system, identifying smooth-transition temperature regions [48, 50, 71, 72]. Using REMD enhances the number of temperatures that can be studied simultaneously, ensuring better ensemble sampling at each temperature. A key challenge in studying elastin-like sequences lies in accurate characterization of a highly dynamic, multi-state system. By performing intensive REMD simulations with read-outs for a large number of temperature data points, we are able to show, for the first time, that the molecular collapse previously described happens as a gradual transition in a well-defined temperature region at the single-molecule scale.

The ELP systems are highly dynamic; therefore, effective sampling, ensured by Replica Exchange simulations, is needed to capture the full range of accessible molecular structures at each temperature. A large spread is observed in molecular size across all temperatures, illustrated by the distribution of radii of gyration across temperatures in SI Figure 11. At high temperatures, the range of accessible structures shrinks by over $15 \%$, with structures 
more densely concentrated around a smaller radius of gyration, manifested by a narrower distribution peak.

As a result of a flat energy landscape traversed by the system even at high temperature, open structures populate the ensemble, though they compose a smaller fraction than at low temperature, evident in the wide distributions of radii of gyration across temperatures (SI

Figure 11). These tools permit the identification of molecular-level detail comparisons across sequences and environmental conditions to shed light on the transition behavior of single-molecule ELP systems, and on ways of enhancing, reducing and suppressing such transitions.

After initial equilibration, subsequent Replica Exchange Molecular Dynamics simulations are carried out in the canonical ensemble. REMD improves sampling compared to classical molecular dynamics (MD) simulations by integrating Monte Carlo exchanges into a classical MD simulation scheme. High temperatures allow for wide conformational space sampling, avoiding protein entrapment in local-minima free energy states via thermal stimulation. Frequent exchanges ensure wide sampling across temperature replicas.

For each system, 60 temperature replicas are used, exponentially distributed from $280 \mathrm{~K}$ to $400 \mathrm{~K}$ [73]. Each replica is simulated for $10 \mathrm{~ns}$, for a total simulation time of $600 \mathrm{~ns}$ across all temperatures. We note that simulation time is scaled with system size, so for systems of much larger size, longer time-scale simulations would be required to fold the protein. Beyond system sizes accessible to classical MD or Replica Exchange MD, coarse graining approaches would be required [74]. Exchanges are attempted after 2 ps equilibration runs, and are accepted according to the Metropolis criterion. Exchange acceptance ratios are between 30-40\%, signifying sufficient sampling. Representative structures are determined by analyzing the ensemble of structures in the final 2 ns of each replica. K-means clustering is used to group structures into clusters according to root mean square deviation of $4 \AA$ for the low-temperature replica at $280 \mathrm{~K}$ and $3.5 \AA$ for the high-temperature replica at $340 \mathrm{~K}$. Representative structures with lowest potential energy are chosen from most populated clusters, represented by significance factor $p$ in SI Figures 2-5. Analysis of representative structures is carried out using the MMTSB script package [75]. All simulations are completed using the Extreme Science and Engineering Discovery Environment (XSEDE) [76].

\section{Analysis of Molecular Structures}

We use the last 8 ns of each REMD run to analyze the molecular structures of ELP sequences. This is done to retain a large structure ensemble while sampling from the equilibrated system. We identify molecular transitions characterized by changes in radius of gyration, hydrogen bonding, solvent accessible surface area, secondary structure and behavior of solvation waters. Radius of gyration gives a measure of the compactness of the structure, defined as

$$
R_{g}=\left(\frac{\sum_{i}\left\|r_{i}\right\|^{2} m_{i}}{\sum_{i} m_{i}}\right)^{\frac{1}{2}}
$$


where $m_{i}$ is the mass of atom $i$ and $\boldsymbol{r}_{i}$ is the position of atom $i$ with respect to the center of mass of the molecule. Protein secondary structure is computed using the DSSP algorithm $[77,78]$. Hydrogen bonds are determined using a geometric definition, with the donorhydrogen-acceptor angle of 30 degrees and a cutoff distance of $0.35 \mathrm{~nm}$ between the donor and the acceptor. To measure hydrogen bond distributions within peptides, we define $\eta$, the hydrogen bond scaled occupancy spread as

$$
\eta=\frac{1}{b} \sum_{i} \sum_{j} O(i, j) \times|I(i)-I(j)|
$$

where $O(i, j)$ is the fraction of time a hydrogen bond exists in the ensemble between donor $i$ and acceptor $j, I(i)$ and $I(j)$ are the donor and acceptor residue indices and $b$ is the total number of donor-acceptor pairs where a hydrogen bond appears. Results for scaled occupancy spread are shown in Figure 4e-g.

Solvent accessible surface area is computed using the double cubic lattice method [79] implemented in Gromacs [66]. Radius of gyration, secondary structure and solvent accessible surface area analysis is done using Gromacs analysis tools [66] and in-house scripts. Hydrogen bond analysis and visualization of molecular models is performed using VMD 1.9.1 [80] and in-house scripts. Average errors are calculated from 400 ps block averages.

\section{DSSP}

DSSP $[77,78]$ calculates hydrogen bond energies based on 3D protein structure, and assigns the most likely class of secondary structure based on hydrogen bond distributions, identifying helix, beta sheet, hydrogen-bonded turn, bend and random coil structure. Coil structures are defined as regions of low curvature, compared to the high curvature of at least $70^{\circ}$ in bend structures. For the sequences studied here, beta sheet and helix structure content is less than $1 \%$; bend structure represents $15-30 \%$ of the secondary structure content; coil structure 48-61\%; and turn structure 14-22\%.

\section{UV-Vis Spectrophotometry}

Lyophilized elastin-like peptides are purchased from Biomatik (Wilmington, Delaware, USA). The UV-Vis spectra of ELP solutions are obtained by a 14DS spectrophotometer equipped with a Peltier temperature controller (Aviv Biomedical, Lakewood, NJ). The 40 $\mathrm{mg} / \mathrm{ml}$ ELP solution is well-mixed and pipetted into $1 \mathrm{~mm}$ path length absorption cuvettes (Hellma USA, Plainview, NY) and allowed to equilibrate at $4^{\circ} \mathrm{C}(277 \mathrm{~K})$ before measurements. To characterize the inverse temperature transition of ELPs, temperature scans are performed at $350 \mathrm{~nm}$ from 3 to $100^{\circ} \mathrm{C}(276 \mathrm{~K}$ to $373 \mathrm{~K})$ at a rate of 2 degrees $/ \mathrm{min}$ and then cooled to $0^{\circ} \mathrm{C}(273 \mathrm{~K})$ at the same rate. Absorbance readings are taken after equilibrating ELP solutions at each desired temperature for $30 \mathrm{~s}$. This equilibration time was selected to guarantee minimum thermal fluctuation during measurements. The turbidity began to change immediately after the temperature increased above the inverse transition temperature based on our observations. The averaging time of each measurement is $10 \mathrm{~s}$ per step. The baseline scans are taken with the solvent and cuvette under the same conditions and subtracted from the sample scans. 


\section{Results}

We compare the effect of temperature for each system by considering the change in radius of gyration (Figure 2a-d). (GVGVP) ${ }_{3}$ and (GVGVP) 6 sequences in $1 \mathrm{M} \mathrm{NaCl}$ show a distinct, gradual transition to a reduced radius of gyration, indicating a shift from extended to contracted structures with increasing temperature (Figure 2a,c). This is consistent with observations of molecular folding upon increasing temperature, representative of singlemolecule inverse temperature transitions $[48,50,71]$ and a shifting equilibrium between folded and extended structure inherent to elastin [41]. For the (GVGVP) $)_{3}$ sequence the structural transition occurs gradually over a 35 degree temperature range (Figure 2a), between $294 \mathrm{~K}$ and $329 \mathrm{~K}$, consistent with experimental studies of similar sequences, where a transition is identified at $24^{\circ} \mathrm{C}$ (i.e. $297 \mathrm{~K}$ ) [16]. The transition region is defined by the intersection of linear fits to regions below, at, and above transition temperatures. Peptide structures for System 1 are shown at $280 \mathrm{~K}$ (Figure 2e) and $340 \mathrm{~K}$ (Figure 2f), and display molecular collapse accompanied by a reorientation of water molecules and restructuring of hydrogen bond networks.

Doubling the chain length of the peptide almost doubles the change in the radius of gyration across the transition range (Figure 2c,g,h). The temperature transition range is marginally lower than that of the shorter sequence (Figure 2a); between $291 \mathrm{~K}$ and $323 \mathrm{~K}$ (Figure 2c). These findings are consistent with experimental work [17, 42] where transitions occur at lower temperatures as the chain length increases, and coacervation propensity is increased with longer chain length [40]. We note that at the single-molecule scale, the main difference between shorter and longer chain length is the magnitude of the transition, or the degree of molecular collapse, rather than a significant shift in the temperature transition region. The change in the radius of gyration for the longer sequence is approximately two times the change in radius of gyration in the shorter sequence. Consequently, the slope of the transition region for the longer sequence indicated by the green line in Figure 2c is about two times as steep as that for the shorter sequence, in Figure 2a. A potential discrepancy with experimental findings where longer chain lengths introduce a pronounced shift to lower transition temperatures, appreciates that at the single-molecule scale, the molecular collapse occurs gradually. Thus, we propose that because the magnitude of this transition, defined here as the change in the radius of gyration, is more significant for longer chain lengths, this system reaches a saturated state inducing a macroscale phase transition at a lower temperature.

In contrast, no significant transition is exhibited by (GVGVP)(GKGVP)(GVGVP) in $1 \mathrm{M} \mathrm{NaCl}$. A single residue substitution from valine $(\mathrm{V})$ to lysine $(\mathrm{K})$ in the seventh position of the (GVGVP) ${ }_{3}$ sequence suppresses a structural transition with temperature (Figure $\mathbf{2 b}$ ). The difference between low- and high- temperature values is not significant: for $280 \mathrm{~K}$ and 340 $\mathrm{K}(p=0.642$ for $\alpha=5 \%)$ and for $280 \mathrm{~K}$ and $380 \mathrm{~K}(p=0.930$ for $\alpha=5 \%)$. The molecular model agrees with experimental findings [16] where a lysine substitution leads to an upshift in the transition temperature from $24^{\circ} \mathrm{C}$ to $120^{\circ} \mathrm{C}(297 \mathrm{~K}$ to $393 \mathrm{~K})$, albeit beyond the temperature range we consider here. Our results match predictions that ELPs containing charged residues would have high transition temperatures inaccessible in aqueous solutions $[16,81]$. 
As sequence modification serves as a trigger for modulating transition temperature, we next turn to consider the effect of changing salt concentration on the molecule's structural integrity. On this basis, a reduction in salt concentration from $1 \mathrm{M}$ to $100 \mathrm{mM} \mathrm{NaCl}$ decreases the magnitude of the structural transition of (GVGVP) 3 (Figure 2d). The difference between low- and high-temperatures is not significant: for $280 \mathrm{~K}$ and $340 \mathrm{~K}$ ( $p=0.447$ for $\alpha=5 \%$ ) and for $280 \mathrm{~K}$ and $380 \mathrm{~K}(p=0.104$ for $\alpha=5 \%)$. We find that higher salt concentration results in ion penetration into the protein core, which likely facilitates the transition observed (Figure $\mathbf{2} \mathbf{i}, \mathbf{j}$ ). Our results are consistent with experimental work showing that higher salt concentration can induce transitions by lowering the transition temperature [17].

To validate our predictions, we conduct a series of experiments and consider the effect of increasing salt concentration to induce a transition in the lysine-containing sequence. First, we confirm with UV spectroscopy that a lysine substitution effectively eliminates a temperature transition (Figure 3a). The turbidity profile of $40 \mathrm{mg} / \mathrm{ml}$ (GVGVP)(GKGVP)(GVGVP) in deionized water at $350 \mathrm{~nm}$ shows no significant change in the temperature range from 293 to $353 \mathrm{~K}$, consistent with a model where the structural transition is suppressed by inserting a charged residue into the elastin sequence. To demonstrate the predictive nature of the model system, we increase the $\mathrm{NaCl}$ concentration of (GVGVP)(GKGVP)(GVGVP) from $0.1 \mathrm{M} \mathrm{NaCl}$, where no transition is observed, to $5 \mathrm{M} \mathrm{NaCl}$, finding a structural transition at $333 \mathrm{~K}$ (Figure $\mathbf{3 b}$ ).

Up to now, we have defined the structural transitions based on the change in the radius of gyration in different sequences. We also consider a number of other parameters to characterize the nature of the transition at the molecular scale. Differences between low and high temperature values for all parameters, including radius of gyration, are listed in SI Table 2. The number of hydrogen bonds (Figure 4a-d) formed within the protein for Systems 1 and 3 increases with temperature, corresponding to the local structural ordering of the peptides (Figure 4a,c). In System 1, the average number of hydrogen bonds present increases from 0.5 to 1 as temperature is raised (Figure 4a). In System 3, this increase is from 2 to 3 hydrogen bonds (Figure 4c). The change in the number of hydrogen bonds is doubled, from 0.5 to 1 , from System 1 to System 3. This is a reasonable result considering that a longer sequence will allow for a greater number of hydrogen bonds to form (SI Table 2). This result is also consistent with the radius of gyration data between the two systems. Peptide-peptide hydrogen bonds (Figure 4a,c) in Systems 1 and 3 replace water-water hydrogen bonds in the nearest solvation shells and peptide-water hydrogen bonds as the structure collapses (SI Figure 6a,c, SI Figure 7a,c). The increased formation of hydrogen bonds within the peptides at higher temperatures is counterintuitive from a kinetic standpoint, and drives structural stabilization. This agrees with findings that suggest the presence of dynamic beta turns that oscillate between disordered and hydrogen bonded states [38], and with a general absence of ordered secondary structure identified through nuclear magnetic resonance [82-84]. Earlier studies additionally point to the joint contributions of glycine $(\mathrm{G})$ and proline $(\mathrm{P})$ to the solvation of the peptide backbone and resultant disordered nature of the peptide, where glycine adds a high degree of chain mobility due to its small size and proline is a rigidity promoter that prohibits the formation of structure-stabilizing hydrogen bonds [51]. 
To determine the relative locations of the hydrogen bonds along the length of the protein, we define $\eta$, or scaled occupancy spread (Equation 2). This measure captures the relative distance between donor and acceptor residues where a hydrogen bond may exist. The scaled occupancy spread almost doubles, from 1 to 2 , across the temperature transition range for Systems 1 to 3, indicating that close-neighbor hydrogen bonds are replaced by hydrogen bonds connecting distant residues as temperature increases (Figure 4e, SI Figure 8a). This is consistent with the observation that the peptide folds up and is then stabilized by forming a hydrogen bond between opposite ends of the molecule. Hydrogen bond occupancy at low temperature ( $280 \mathrm{~K}$ ) for sequence (GVGVP) 3 is shown in Figure $\mathbf{4 f}$. The occupancy indicates the fraction of time that a particular hydrogen bond exists between a donor-acceptor pair. The highest occurrence of hydrogen bonds appears along the diagonal of donor-acceptor residue pairs at low temperature, implying that the majority of hydrogen bonds that can form are between nearest neighbor residues. At high temperature $(340 \mathrm{~K})$, the distribution of hydrogen bond occupancy shifts away from the diagonal of donor-acceptor residue pairs, as illustrated by the difference in occupancy between high and low temperatures in Figure 4g. This shift indicates a change in the distribution of the locations of hydrogen bonds that can form. At higher temperatures, hydrogen bonds tend to form between residues that are farther apart than nearest neighbors, which is the trend seen at low temperature. This trend is not observed for Systems 2 and 4 (SI Figure 8b,c). The shift in hydrogen bond position for Systems 1 and 3 may be responsible for stabilizing structures at higher temperatures. Indeed, we find that the standard deviation of the radius of gyration is lower at high temperatures for Systems 1 and 3, and to a lower extent for System 4, indicating less variance in structure and greater stability at increased temperatures (SI Figure 9). This counterintuitive result from a kinetics standpoint highlights the unique inverse nature of ELP temperature transitions.

Trends in the amount of solvent accessible surface area across the temperature range (Figure 5a-d) are consistent with patterns in radius of gyration: when structural folding occurs, it is accompanied by a reduced solvent accessible surface area. This is clearly visible for Systems 1 and 3 (Figure 5a,c), whereas no shift is detected for System 2 (Figure 5b). For System 4, a mild shift towards a reduced solvent accessible surface area is observed at a higher temperature than for System 1 (Figure 5d). The patterns observed in solvation water molecules around the protein are similarly consistent (Figure 5e-h). The first hydration shell in each system occurs approximately within $2.1 \AA$ Af the protein, indicated by the first local minimum in the protein-water radial distribution functions (SI Figure 10). After the first local minima, a mild second local minima is discerned at approximately $3 \AA$. Beyond the first two hydration shells, water molecules are arranged as in bulk, as indicated by the flattening of the radial distribution functions, as a result of the small size of the protein that does not significantly disturb the bulk water. Because there is a constant transfer of molecules across the boundary of the first solvation shell, we consider a shell of $3 \AA$ for solvation water calculations to capture the behavior of water molecules at the protein's surface. We observe that the radial distribution function peaks flatten with temperature, most significantly for Systems 1 and 3 that display transitions, indicating that water molecules in the local hydration shells are replaced by bulk water as temperature increases. The intimate link between the orientation and distribution of water molecules facilitating elastin transitions is consistent with elastin's high degree of sensitivity to levels of hydration [46]. 
The structural collapse and associated reduction in the solvent accessible surface area is driven by changes in local secondary structure (Figure 5i-1). These changes are characterized by a distinct transition from coil to turn structures, defined by the DSSP (Define Secondary Structure of Proteins) algorithm [77, 78]. In Systems 1 and 3, with increasing temperature, coils are replaced by ordered secondary structure, including a very low percentage $(<1 \%)$ of sheet and helix, but primarily hydrogen-bonded turns (Figure $\mathbf{5 i}, \mathbf{k})$. We note that mild changes in secondary structure are observed for Systems 2 and 4 (Figure 5j,l). This observation indicates that at the local level, these sequences exhibit some structural folding, though a full molecular collapse is not observed. In System 4, the transition shifts to higher temperatures (Figure 5l), consistent with experimental findings in this study and earlier work $[17,85]$. Overall, our findings are compatible with work on other forms of elastin, including other kinds of ELPs and purified elastin. For example, studies on VGGVG by Tamburro and coworkers [86, 87] and studies on purified elastin by Samouillan et. al. [88] indicate a presence of an equilibrium between flexible and ordered zones, exhibiting a variety of conformational preferences.

\section{Salt Concentration Effects}

Based on the preceding discussion, we summarize the main molecular effects on structural transition in ELPs brought on by changing salt concentration. The evident transition at higher salt concentration of System 1, in $1 \mathrm{M} \mathrm{NaCl}$, compared to System 4, in $100 \mathrm{mM} \mathrm{NaCl}$, observed as the change in radius of gyration (Figure 2a,d), is attributed to an increased interaction between ions, protein and water molecules in the nearest hydration shells. The presence of ions in the first two nearest hydration shells increases noticeably at higher $\mathrm{NaCl}$ concentration in System 1. On average, 1.8 sodium and chloride ions exist in a $3 \AA$ solvation shell around the protein in $1 \mathrm{M} \mathrm{NaCl}$, in contrast to 0.5 ions in $100 \mathrm{mM} \mathrm{NaCl}$. This is consistent across all temperatures. The ions that diffuse into the nearest hydration shell of the protein can form strong interactions with electronegative atoms in the peptide chain, facilitating structural folding (Figure 2i,j). Moreover, ions act to disturb the hydrogenbonded water network surrounding the protein, promoting the formation of hydrogen bonds within the peptide (Figure 4a) as solvation waters are expelled from the nearest hydration shell (Figure 5e).

\section{Chain Length Effects}

Summarizing the effects discussed upon increasing chain length, we find that as the chain length is increased, the expanded form of the molecule expels an increased number of solvation waters upon folding. In System 1, the number of water molecules in a $3 \AA$ solvation shell reduces from 68 to 65 , with increasing temperature, compared to a change from 117 to 110 water molecules in System 3 (Figure 5e,g). Water-water and peptidewater hydrogen bonds break, ejecting water molecules trapped at the surface of the protein out of the solvation shell into the bulk. As the water molecules shift in the longer-length peptide, it folds over itself, forming turn secondary structure (Figure 5k) stabilized by hydrogen bonds (Figure 4c).

\section{Chemistry Effects}


Amino acid substitutions can substantially shift the transition temperatures of ELPs [16, 17]. At the molecular level, we find that transitions in local hydrogen bonding and secondary structure, as well as any structural peptide collapse are absent for the lysinecontaining sequence. To evaluate these effects we consider the local environment in a $3 \AA$ radius solvation shell around the seventh amino acid in ELP Systems 1 and 2. In System 1, the seventh residue is valine, while in System 2 it is replaced by lysine. Notably, this valine in System 1 contains approximately 6 water molecules in a $3 \AA$ radius solvation shell, compared to approximately 10 water molecules surrounding the lysine residue in System 2 (Figure 6a,b). In both Systems 1 and 2 the change in solvation waters around the seventh residue is minimal - a difference of $<1$ solvation water molecule between high and low temperatures - compared to the raw number of water molecules surrounding the seventh residue in each system ( 6 water molecules in System 1 and 10 molecules in System 2).

We can understand this result by considering the longer side chain of lysine compared to that of valine, a correspondingly larger exposed surface area to water and exposed positive charge. At high temperatures, both sequences exhibit a loss in water - water hydrogen bonds in the $3 \AA$ A radius solvation shell around the seventh residue (Figure 6c,d), as well as a decrease in seventh residue - water hydrogen bonds (Figure 6e,f). We see an overall greater number of water-water and seventh residue-water hydrogen bonds in the lysinecontaining sequence (Figure 6d,f) compared to the valine-containing sequence (Figure 6e,c). For example, at $280 \mathrm{~K}$, System 2 contains 2.5 water-water hydrogen bonds in the $3 \AA$ radius solvation shell around the seventh lysine (Figure $\mathbf{6 d}$ ) and 3.5 lysine-water hydrogen bonds (Figure 6f), compared to, in System 1, 1.6 water-water hydrogen bonds in the $3 \AA$ radius solvation shell around the seventh valine (Figure $6 \mathbf{c}$ ) and 1.4 valine-water hydrogen bonds (Figure 6e). Indeed, at high temperature, the number of hydrogen bonds involving the seventh lysine in System 2 exceeds the number of hydrogen bonds involving the seventh valine in System 1 - even at low temperature. Specifically, at $400 \mathrm{~K}$, there are 2.8 lysine-water hydrogen bonds in System 2 (Figure 6f) compared to 1.4 valine-water hydrogen bonds in System 1 at $280 \mathrm{~K}$ (Figure 6e). This suggests that the lysine residue in System 2 acts as a stiffened hinge that is densely hydrogen-bonded to an interconnected network of water molecules. The presence of a larger number of interconnected waters enveloping the center of the peptide chain prevents a structural collapse representative of the transition in System 1. This is supported by the fact that the number of hydrogen bonds between the peptide and the seventh valine in System 1 increases modestly, while no increase is observed for lysine (Figure $\mathbf{6 g}, \mathbf{h}$ ).

Remarkably, the local effects around the guest residue in the seventh position explain the temperature-induced collapse of System 1, supported by the formation of hydrogen bonds between distant residues in the peptide chain (GVGVP) 3 . The small, flexible valine hinge in System 1 allows for structural collapse of the molecule and consequent system stabilization (Figure 6i), in contrast to the lysine-containing System 2 (Figure 6j). We recognize two disparate roles for water: it acts as a plasticizer, enhancing the conformational mobility of the peptide [38]; yet, simultaneously, it provides an obstacle to structural flexibility based on its interaction with local components of the peptide.

\section{Conclusion}


In summary, in this study the effects of ionic concentration, chain length, and sequence chemistry on temperature-induced structural transitions in elastin-like peptides are presented. We consider these systems at the single-molecule scale finding that structural transitions occur as smooth shifts within defined transition regions. The behavior of water networks around the peptide is a driving factor in structural transitions. In the presence of higher salt concentration, we find that ions can more easily diffuse into the nearest hydration shell of the protein, forming strong interactions with electronegative atoms in the peptide chain, which facilitates structural folding. Ions act to disturb the hydrogenbonded water network surrounding the protein, promoting the formation of hydrogen bonds within the peptide as solvation waters are expelled from the nearest hydration shell. Longer chain length drives the expulsion of solvation waters as temperature is increased. As water-water and peptide-water hydrogen bonds brake, water molecules trapped at the surface of the protein are pushed out of the solvation shell into the bulk. This introduces the formation of turns stabilized by hydrogen bonds and an overall shift to compact molecular structure. Finally, sequence chemistry changes influence the local interactions with water. We compare a lysine-for-valine substitution and find that the lysine residue stiffens the hinge by hydrogen-bonding to an interconnected network of water molecules, and so prevents a structural collapse. We find that water acts as a plasticizer, enhancing the conformational mobility of the peptide [38] and simultaneously reducing structural flexibility based on its interactions with local components of the peptide.

\section{Acknowledgements}

We thank Zhao Qin and Davoud Ebrahimi for fruitful discussions. This work used the Extreme Science and Engineering Discovery Environment (XSEDE), supported by the National Science Foundation grant number ACI-1053575. The authors acknowledge support from the NIH (5U01EB014976 and EB014283), ONR (N000141612333) and AFOSR (FA9550-11-1-0199).

\section{References}

[1] J. Lee, C. W. Macosko, and D. W. Urry, "Phase transition and elasticity of proteinbased hydrogels," Journal Of Biomaterials Science. Polymer Edition, vol. 12, pp. 229242, 2001.

[2] D. W. Urry, "Entropic elastic processes in protein mechanisms. I. Elastic structure due to an inverse temperature transition and elasticity due to internal chain dynamics," J Protein Chem, vol. 7, pp. 1-34, Feb 1988.

[3] D. W. Urry, R. G. Shaw, and K. U. Prasad, "Polypentapeptide of elastin: temperature dependence of ellipticity and correlation with elastomeric force," Biochemical And Biophysical Research Communications, vol. 130, pp. 50-57, 1985.

[4] D. W. Urry, T. L. Trapane, M. Iqbal, C. M. Venkatachalam, and K. U. Prasad, "Carbon13 NMR relaxation studies demonstrate an inverse temperature transition in the elastin polypentapeptide," Biochemistry, vol. 24, pp. 5182-5189, 1985.

[5] D. W. Urry, T. L. Trapane, and K. U. Prasad, "Phase-structure transitions of the elastin polypentapeptide-water system within the framework of composition-temperature studies," Biopolymers, vol. 24, p. 2345, 12// 1985. 
[6] D. W. Urry, "Entropic elastic processes in protein mechanisms. II. Simple (passive) and coupled (active) development of elastic forces," J Protein Chem, vol. 7, pp. 81114, Apr 1988.

[7] D. W. Urry, "Free energy transduction in polypeptides and proteins based on inverse temperature transitions," Progress In Biophysics And Molecular Biology, vol. 57, pp. 23-57, 1992.

[8] D. W. Urry, "MOLECULAR MACHINES - HOW MOTION AND OTHER FUNCTIONS OF LIVING ORGANISMS CAN RESULT FROM REVERSIBLE CHEMICAL-CHANGES," ANGEWANDTE CHEMIE-INTERNATIONAL EDITION, vol. 32, pp. 819-841, 1993.

[9] D. W. Urry, What sustains life? [electronic resource] : consilient mechanisms for protein-based machines and materials: New York, N.Y. : Springer, c2006., 2006.

[10] S. Roberts, M. Dzuricky, and A. Chilkoti, "Review: Elastin-like polypeptides as models of intrinsically disordered proteins," FEBS Letters, vol. 589, pp. 2477-2486, 9/14/14 September 20152015.

[11] J. R. McDaniel, D. C. Radford, and A. Chilkoti, "A Unified Model for De Novo Design of Elastin-like Polypeptides with Tunable Inverse Transition Temperatures," BIOMACROMOLECULES, vol. 14, pp. 2866-2872, 2013.

[12] D. Kurzbach, W. Hassouneh, J. R. McDaniel, E. A. Jaumann, A. Chilkoti, and D. Hinderberger, "Hydration layer coupling and cooperativity in phase behavior of stimulus responsive peptide polymers," J Am Chem Soc, vol. 135, pp. 11299-308, Jul 312013.

[13] G. C. Yeo, F. W. Keeley, and A. S. Weiss, "Coacervation of tropoelastin," Advances in Colloid and Interface Science, vol. 167, pp. 94-103, 1/1/2011 2011.

[14] D. W. Urry, "Physical chemistry of biological free energy transduction as demonstrated by elastic protein-based polymers," JOURNAL OF PHYSICAL CHEMISTRY B, vol. 101, pp. 11007-11028, 1997.

[15] D. W. Urry, "Five axioms for the functional design of peptide-based polymers as molecular machines and materials: Principle for macromolecular assemblies," BIOPOLYMERS, vol. 47, pp. 167-178, 1998.

[16] D. W. Urry, D. C. Gowda, T. M. Parker, C. H. Luan, M. C. Reid, C. M. Harris, et al., "Hydrophobicity scale for proteins based on inverse temperature transitions," Biopolymers, vol. 32, pp. 1243-1250, 1992.

[17] H. Nuhn and H. A. Klok, "Secondary Structure Formation and LCST Behavior of Short Elastin-Like Peptides," BIOMACROMOLECULES, vol. 9, pp. 2755-2763, 2008.

[18] A. Ribeiro, F. J. Arias, J. Reguera, M. Alonso, and J. C. Rodríguez-Cabello, "Article: Influence of the Amino-Acid Sequence on the Inverse Temperature Transition of Elastin-Like Polymers," Biophysical Journal, vol. 97, pp. 312-320, 7/8/8 July 2009 2009.

[19] D. E. Meyer and A. Chilkoti, "Genetically encoded synthesis of protein-based polymers with precisely specified molecular weight and sequence by recursive directional ligation: Examples from the elastin-like polypeptide system," BIOMACROMOLECULES, vol. 3, pp. 357-367, 2002.

[20] A. Girotti, J. Reguera, F. J. Arias, M. Alonso, A. M. Testera, and J. C. Rodriguez-Cabello, "Influence of the molecular weight on the inverse temperature transition of a model genetically engineered elastin-like pH-responsive polymer," MACROMOLECULES, vol. 37, pp. 3396-3400, 2004.

[21] J. Reguera, D. W. Urry, T. M. Parker, D. T. McPherson, and J. C. Rodriguez-Cabello, "Effect of $\mathrm{NaCl}$ on the exothermic and endothermic components of the inverse 
temperature transition of a model elastin-like polymer," BIOMACROMOLECULES, vol. 8, pp. 354-358, 2007.

[22] J. A. MacKay, D. J. Callahan, K. N. FitzGerald, and A. Chilkoti, "Quantitative Model of the Phase Behavior of Recombinant pH-Responsive Elastin-Like Polypeptides," BIOMACROMOLECULES, vol. 11, pp. 2873-2879, 2010.

[23] L. A. Strzegowski and M. B. Martinez, "Photomodulation of the inverse temperature transition of a modified elastin poly(pentapeptide)," Journal of the American Chemical Society, vol. 116, p. 813, 1994.

[24] M. R. Dreher, A. J. Simnick, K. Fischer, R. J. Smith, A. Patel, M. Schmidt, et al., "Temperature triggered self-assembly of polypeptides into multivalent spherical micelles," JOURNAL OF THE AMERICAN CHEMICAL SOCIETY, vol. 130, pp. 687-694, 2008.

[25] J. R. McDaniel, I. Weitzhandler, S. Prevost, K. B. Vargo, M.-S. Appavou, D. A. Hammer, et al., "Noncanonical self-assembly of highly asymmetric genetically encoded polypeptide amphiphiles into cylindrical micelles," Nano Letters, vol. 14, pp. 65906598, 2014.

[26] K. Trabbic-Carlson, L. A. Setton, and A. Chilkoti, "Swelling and mechanical behaviors of chemically cross-linked hydrogels of elastin-like polypeptides,"

BIOMACROMOLECULES, vol. 4, pp. 572-580, 2003.

[27] L. Martín, M. Alonso, A. Girotti, F. J. Arias, and J. C. Rodríguez-Cabello, "Synthesis and characterization of macroporous thermosensitive hydrogels from recombinant elastin-like polymers," Biomacromolecules, vol. 10, pp. 3015-3022, 2009.

[28] S. R. MacEwan and A. Chilkoti, "Review: Applications of elastin-like polypeptides in drug delivery," Journal of Controlled Release, vol. 190, pp. 314-330, 9/28/28 September 20142014.

[29] J. R. McDaniel, D. J. Callahan, and A. Chilkoti, "Drug delivery to solid tumors by elastin-like polypeptides," Advanced Drug Delivery Reviews, vol. 62, pp. 1456-1467, $1 / 1 / 20102010$.

[30] R. Herrero-Vanrell, A. C. Rincón, M. Alonso, V. Reboto, I. T. Molina-Martinez, and J. C. Rodríguez-Cabello, "Self-assembled particles of an elastin-like polymer as vehicles for controlled drug release," Journal of Controlled Release, vol. 102, pp. 113-122, $1 / 1 / 20052005$.

[31] D. L. Nettles, A. Chilkoti, and L. A. Setton, "Applications of elastin-like polypeptides in tissue engineering," Advanced Drug Delivery Reviews, vol. 62, pp. 1479-1485, $1 / 1 / 20102010$.

[32] S. R. MacEwan and A. Chilkoti, "Digital Switching of Local Arginine Density in a Genetically Encoded Self-Assembled Polypeptide Nanoparticle Controls Cellular Uptake," NANO LETTERS, vol. 12, pp. 3322-3328, 2012.

[33] M. R. Dreher, W. G. Liu, C. R. Michelich, M. W. Dewhirst, and A. Chilkoti, "Thermal cycling enhances the accumulation of a temperature-sensitive biopolymer in solid tumors," CANCER RESEARCH, vol. 67, pp. 4418-4424, 2007.

[34] M. Amiram, K. M. Luginbuhl, X. H. Li, M. N. Feinglos, and A. Chilkoti, "Injectable protease-operated depots of glucagon-like peptide-1 provide extended and tunable glucose control," PROCEEDINGS OF THE NATIONAL ACADEMY OF SCIENCES OF THE UNITED STATES OF AMERICA, vol. 110, pp. 2792-2797, 2013.

[35] D. E. Meyer, B. C. Shin, G. A. Kong, M. W. Dewhirst, and A. Chilkoti, "Drug targeting using thermally responsive polymers and local hyperthermia," Journal of Controlled Release, vol. 74, pp. 213-224, Jul 62001. 
[36] M. R. Dreher, D. Raucher, N. Balu, O. Michael Colvin, S. M. Ludeman, and A. Chilkoti, "Evaluation of an elastin-like polypeptide-doxorubicin conjugate for cancer therapy," J Control Release, vol. 91, pp. 31-43, Aug 282003.

[37] C. L. DW Urry, TM Parker, DC Gowda, KU Prasad, MC Reid, A Safavy, "Temperature of Polypeptide Inverse Temperature Transition Depends on Mean Residue Hydrophobicity," Journal of the American Chemical Society, vol. 113, pp. 4346-4348, 1991.

[38] L. Debelle and A. M. Tamburro, "Elastin: molecular description and function," INTERNATIONAL JOURNAL OF BIOCHEMISTRY \& CELL BIOLOGY, vol. 31, pp. 261272, 1999.

[39] A. M. Tamburro, B. Bochicchio, and A. Pepe, "Dissection of human tropoelastin: exonby-exon chemical synthesis and related conformational studies," Biochemistry, vol. 42, pp. 13347-62, Nov 182003.

[40] A. Pepe, D. Guerra, B. Bochicchio, D. Quaglino, D. Gheduzzi, I. Pasquali Ronchetti, et al., "Dissection of human tropoelastin: supramolecular organization of polypeptide sequences coded by particular exons," Matrix Biol, vol. 24, pp. 96-109, Apr 2005.

[41] A. M. Tamburro, B. Bochicchio, and A. Pepe, "The dissection of human tropoelastin: from the molecular structure to the self-assembly to the elasticity mechanism," Pathol Biol (Paris), vol. 53, pp. 383-9, Sep 2005.

[42] D. E. Meyer and A. Chilkoti, "Quantification of the effects of chain length and concentration on the thermal behavior of elastin-like polypeptides," BIOMACROMOLECULES, vol. 5, pp. 846-851, 2004.

[43] R. Herrero-Vanrell, A. C. Rincon, M. Alonso, V. Reboto, I. T. Molina-Martinez, and J. C. Rodriguez-Cabello, "Self-assembled particles of an elastin-like polymer as vehicles for controlled drug release," J Control Release, vol. 102, pp. 113-22, Jan 202005.

[44] A. Chilkoti, M. R. Dreher, and D. E. Meyer, "Design of thermally responsive, recombinant polypeptide carriers for targeted drug delivery," Adv Drug Deliv Rev, vol. 54, pp. 1093-111, Oct 182002.

[45] J. Dandurand, V. Samouillan, C. Lacabanne, A. Pepe, and B. Bochicchio, "Water structure and elastin-like peptide aggregation A differential calorimetric approach," Journal of Thermal Analysis and Calorimetry, vol. 120, pp. 419-426, Apr 2015.

[46] V. Samouillan, C. Andre, J. Dandurand, and C. Lacabanne, "Effect of water on the molecular mobility of elastin," Biomacromolecules, vol. 5, pp. 958-64, May-Jun 2004.

[47] C. Sun, O. Mitchell, J. Huang, and G. S. Boutis, "NMR studies of localized water and protein backbone dynamics in mechanically strained elastin," J Phys Chem B, vol. 115, pp. 13935-42, Dec 012011.

[48] B. Li, D. O. V. Alonso, and V. Daggett, "The molecular basis for the inverse temperature transition of elastin," JOURNAL OF MOLECULAR BIOLOGY, vol. 305, pp. 581-592, 2001.

[49] B. Li, D. O. V. Alonso, and V. Daggett, "Stabilization of globular proteins via introduction of temperature-activated elastin-based switches," Structure, vol. 10, pp. 989-998, Jul 2002.

[50] R. Rousseau, E. Schreiner, A. Kohlmeyer, and D. Marx, "Temperature-dependent conformational transitions and hydrogen-bond dynamics of the elastin-like octapeptide GVG(VPGVG): a molecular-dynamics study," Biophysical Journal, vol. 86, pp. 1393-1407, 2004. 
[51] S. Rauscher, S. Baud, M. Miao, F. W. Keeley, and R. Pomes, "Proline and glycine control protein self-organization into elastomeric or amyloid fibrils," STRUCTURE, vol. 14, pp. 1667-1676, 2006.

[52] B. Bochicchio, A. Pepe, F. Delaunay, M. Lorusso, S. Baud, and M. Dauchez, "Amyloidogenesis of proteolytic fragments of human elastin," Rsc Advances, vol. 3, pp. 13273-13285, 2013.

[53] C. Blanchevoye, N. Floquet, A. Scandolera, S. Baud, P. Maurice, O. Bocquet, et al., "Interaction between the Elastin Peptide VGVAPG and Human Elastin Binding Protein," Journal of Biological Chemistry, vol. 288, pp. 1317-1328, Jan 112013.

[54] N. Floquet, S. Hery-Huynh, M. Dauchez, P. Derreumaux, A. M. Tamburro, and A. J. Alix, "Structural characterization of VGVAPG, an elastin-derived peptide," Biopolymers, vol. 76, pp. 266-80, 2004.

[55] J. C. Rodriguez-Cabello, M. Alonso, M. I. Diez, M. I. Caballero, and M. M. Herguedas, "Structural investigation of the poly(pentapeptide) of elastin, poly(GVGVP), in the solid state," Macromolecular Chemistry and Physics, vol. 200, pp. 1831-1838, Aug 1999.

[56] P. C. Gross, W. Possart, and M. Zeppezauer, "An alternative structure model for the polypentapeptide in elastin," Zeitschrift Fur Naturforschung C-a Journal of Biosciences, vol. 58, pp. 873-878, Nov-Dec 2003.

[57] C. Dicko, D. Knight, J. M. Kenney, and F. Vollrath, "Secondary structures and conformational changes in flagelliform, cylindrical, major, and minor ampullate silk proteins. Temperature and concentration effects," Biomacromolecules, vol. 5, pp. 2105-15, Nov-Dec 2004.

[58] O. T. Ukpebor, A. Shah, E. Bazov, and G. S. Boutis, "Inverse temperature transition of elastin like motifs in major ampullate dragline silk: MD simulations of short peptides and NMR studies of water dynamics," Soft Matter, vol. 10, pp. 773-85, Feb 072014.

[59] G. Li, Y. Li, G. Chen, J. He, Y. Han, X. Wang, et al., "Silk-based biomaterials in biomedical textiles and fiber-based implants," Adv Healthc Mater, vol. 4, pp. 113451, Jun 032015.

[60] E. S. Gil and S. M. Hudson, "Stimuli-reponsive polymers and their bioconjugates," Progress in Polymer Science, vol. 29, pp. 1173-1222, Dec 2004.

[61] B. R. Brooks, R. E. Bruccoleri, B. D. Olafson, D. J. States, S. Swaminathan, and M. Karplus, "CHARMM: a program for macromolecular energy, minimisation, and dynamics calculations," Journal of Computational Chemistry, vol. 4, pp. 187-217, 1983.

[62] A. D. MacKerell, D. Bashford, M. Bellott, R. L. Dunbrack, J. D. Evanseck, M. J. Field, et al., "All-Atom Empirical Potential for Molecular Modeling and Dynamics Studies of Proteins," The Journal of Physical Chemistry B, vol. 102, pp. 3586-3616, 1998/04/01 1998.

[63] S. W. Chang, B. P. Flynn, J. W. Ruberti, and M. J. Buehler, "Molecular mechanism of force induced stabilization of collagen against enzymatic breakdown," Biomaterials, vol. 33, pp. 3852-3859, May 2012.

[64] A. Gautieri, M. I. Pate, S. Vesentini, A. Redaelli, and M. J. Buehler, "Hydration and distance dependence of intermolecular shearing between collagen molecules in a model microfibril," Journal of Biomechanics, vol. 45, pp. 2079-2083, Aug 92012.

[65] M. Baer, E. Schreiner, A. Kohlmeyer, R. Rousseau, and D. Marx, "Inverse temperature transition of a biomimetic elastin model: Reactive flux analysis of folding/unfolding 
and its coupling to solvent dielectric relaxation," Journal of Physical Chemistry B, vol. 110, pp. 3576-3587, Mar 22006.

[66] D. Van Der Spoel, E. Lindahl, B. Hess, G. Groenhof, A. E. Mark, and H. J. Berendsen, "GROMACS: fast, flexible, and free," J Comput Chem, vol. 26, pp. 1701-18, Dec 2005.

[67] Y. Sugita and Y. Okamoto, "Replica-exchange molecular dynamics method for protein folding," Chemical Physics Letters, vol. 314, pp. 141-151, 11/26/ 1999.

[68] H. J. C. Berendsen, J. P. M. Postma, W. F. van Gunsteren, A. DiNola, and J. R. Haak, "Molecular dynamics with coupling to an external bath," Journal of Chemical Physics, vol. 81, p. 3684, 10/15/ 1984.

[69] M. Parrinello and A. Rahman, "Polymorphic transitions in single crystals: A new molecular dynamics method," Journal of Applied Physics, vol. 52, p. 7182, 12// 1981.

[70] U. Essmann, L. Perera, M. L. Berkowitz, T. Darden, H. Lee, and L. G. Pedersen, "A smooth particle mesh Ewald method," Journal of Chemical Physics, vol. 103, p. 8577, 1995.

[71] E. Schreiner, C. Nicolini, B. Ludolph, R. Ravindra, N. Otte, A. Kohlmeyer, et al., "Folding and unfolding of an elastinlike oligopeptide: "Inverse temperature Transition," reentrance, and hydrogen-bond dynamics," Physical Review Letters, vol. 92, pp. 148101/1-4, 2004.

[72] N. K. Li, F. García Quiroz, C. K. Hall, A. Chilkoti, and Y. G. Yingling, "Molecular description of the LCST behavior of an elastin-like polypeptide," Biomacromolecules, vol. 15, pp. 3522-3530, 2014.

[73] A. Patriksson and D. van der Spoel, "A temperature predictor for parallel tempering simulations," Physical Chemistry Chemical Physics, vol. 10, pp. 2073-2077, 2008.

[74] M. Seo, S. Rauscher, R. Pomes, and D. P. Tieleman, "Improving Internal Peptide Dynamics in the Coarse-Grained MARTINI Model: Toward Large-Scale Simulations of Amyloid- and Elastin-like Peptides," Journal of Chemical Theory and Computation, vol. 8, pp. 1774-1785, May 2012.

[75] M. Feig, J. Karanicolas, and I. I. I. C. L. Brooks, "MMTSB Tool Set: enhanced sampling and multiscale modeling methods for applications in structural biology," Journal of Molecular Graphics and Modelling, vol. 22, pp. 377-395, 1/1/2004 2004.

[76] J. Towns, T. Cockerill, M. Dahan, I. Foster, K. Gaither, A. Grimshaw, et al., "XSEDE: Accelerating Scientific Discovery," Computing in Science \& Engineering, vol. 16, pp. 62-74, 2014.

[77] R. P. Joosten, T. A. H. te Beek, E. Krieger, M. L. Hekkelman, R. W. W. Hooft, R. Schneider, et al., "A series of PDB related databases for everyday needs," Nucleic Acids Research, vol. 39, pp. D411-D419, 2011.

[78] W. Kabsch and C. Sander, "Dictionary of protein secondary structure: Pattern recognition of hydrogen-bonded and geometrical features," Biopolymers, vol. 22, pp. 2577-2637, 1983.

[79] F. Eisenhaber, P. Lijnzaad, P. Argos, C. Sander, and M. Scharf, "The double cubic lattice method: Efficient approaches to numerical integration of surface area and volume and to dot surface contouring of molecular assemblies," Journal of Computational Chemistry, vol. 16, pp. 273-284, 1995.

[80] W. Humphrey, A. Dalke, and K. Schulten, "VMD: Visual molecular dynamics," Journal of Molecular Graphics, vol. 14, 1996.

[81] T. Christensen, W. Hassouneh, K. Trabbic-Carlson, and A. Chilkoti, "Predicting transition temperatures of elastin-like polypeptide fusion proteins," Biomacromolecules, vol. 14, pp. 1514-1519, 2013. 
[82] M. Hong, D. Isailovic, R. A. McMillan, and V. P. Conticello, "Structure of an elastinmimetic polypeptide by solid-state NMR chemical shift analysis," BIOPOLYMERS, vol. 70, pp. 158-168, 2003.

[83] D. Kurkova, J. Kriz, P. Schmidt, J. Dybal, J. C. Rodriguez-Cabello, and M. Alonso, "Structure and dynamics of two elastin-like polypentapeptides studied by NMR spectroscopy," BIOMACROMOLECULES, vol. 4, pp. 589-601, 2003.

[84] M. S. Pometun, E. Y. Chekmenev, and R. J. Wittebort, "Quantitative observation of backbone disorder in native elastin," JOURNAL OF BIOLOGICAL CHEMISTRY, vol. 279, pp. 7982-7987, 2004.

[85] C. H. Luan, T. M. Parker, K. U. Prasad, and D. W. Urry, "Differential scanning calorimetry studies of $\mathrm{NaCl}$ effect on the inverse temperature transition of some elastin-based polytetra-, polypenta-, and polynonapeptides," Biopolymers, vol. 31, pp. 465-475, 1991.

[86] A. M. Tamburro, V. Guantieri, L. Pandolfo, and A. Scopa, "Synthetic fragments and analogues of elastin. II. Conformational studies," Biopolymers, vol. 29, pp. 855-70, Mar-Apr 1990.

[87] F. Lelj, A. M. Tamburro, V. Villani, P. Grimaldi, and V. Guantieri, "Molecular dynamics study of the conformational behavior of a representative elastin building block: BocGly-Val-Gly-Gly-Leu-OMe," Biopolymers, vol. 32, pp. 161-72, Feb 1992.

[88] V. Samouillan, A. Lamure, E. Maurel, C. Lacabanne, and W. Hornebeck, "Alterations in the chain dynamics of insoluble elastin upon proteolysis by serine elastases,"

Biopolymers, vol. 58, pp. 175-85, Feb 2001. 
Figures and Figure Captions

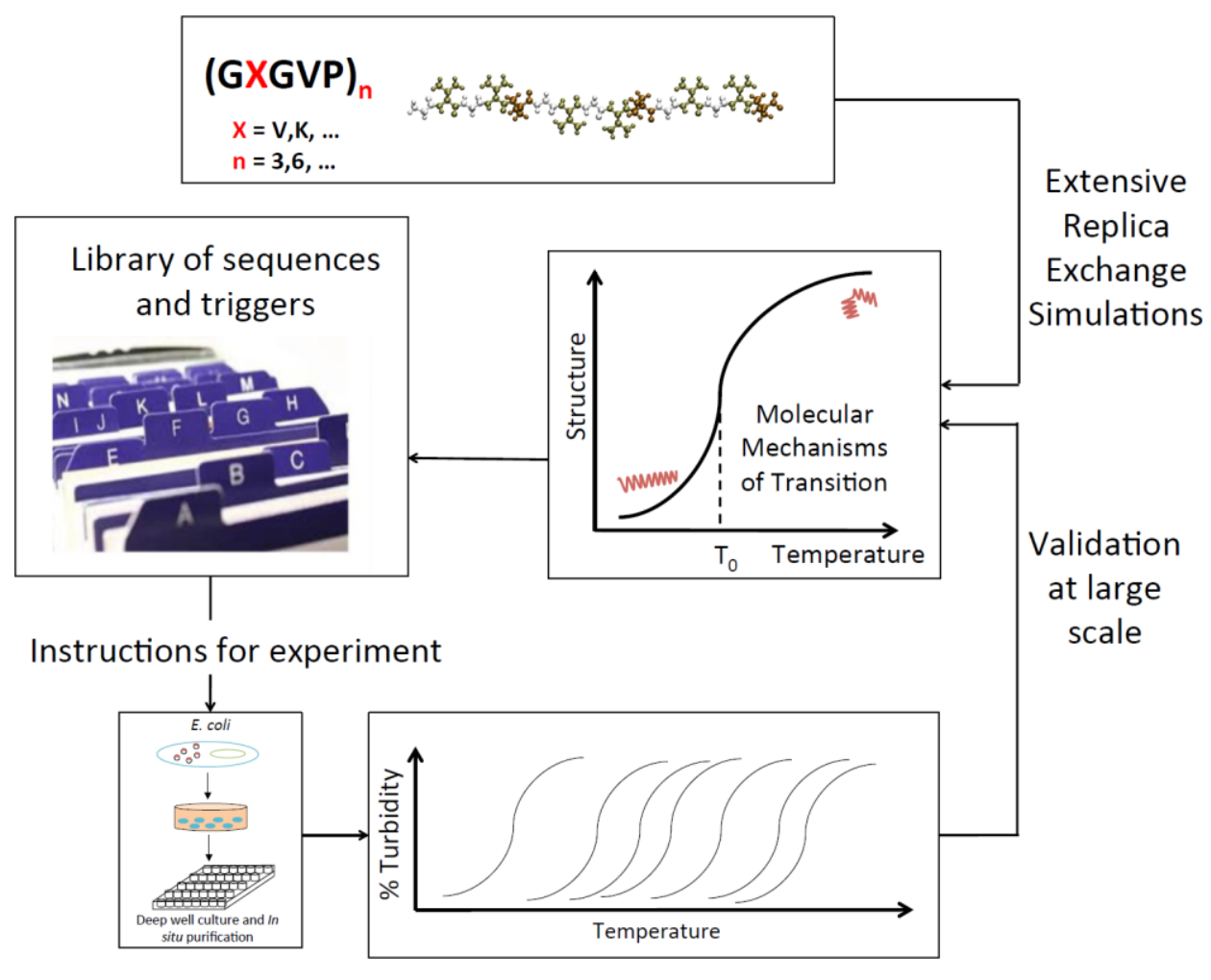

Experimental measurement

Figure 1 | Simulation-experiment material design paradigm flow-chart. Sequences of the form [(GVGVP)(GXGVP)(GVGVP) $]_{\mathrm{n}}$ are simulated with extensive Replica Exchange Molecular Dynamics simulations to identify molecular mechanisms of structural transitions. This yields a basis for a library of available sequences to instruct experimental design. Recombinant DNA technology is used to synthesize proteins in vitro, feeding back into the simulation-experiment design loop. 

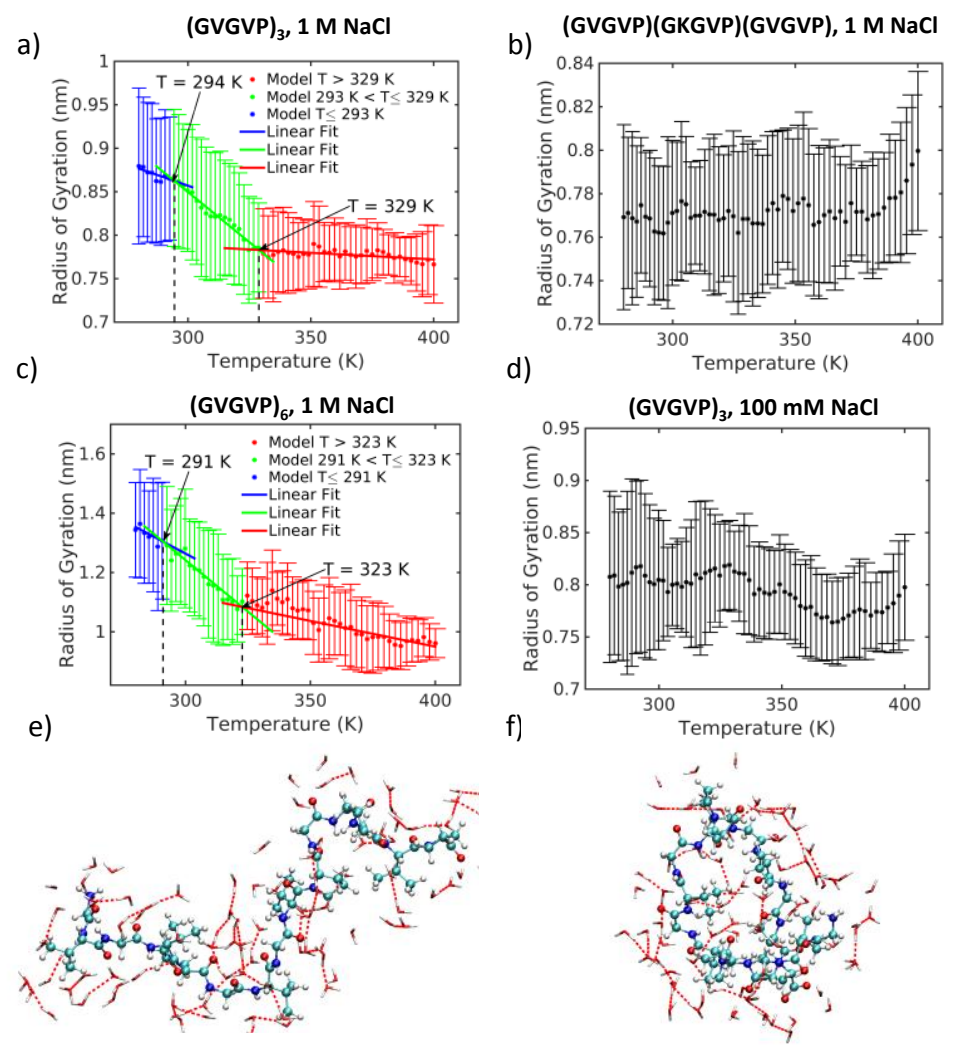

g)

h)
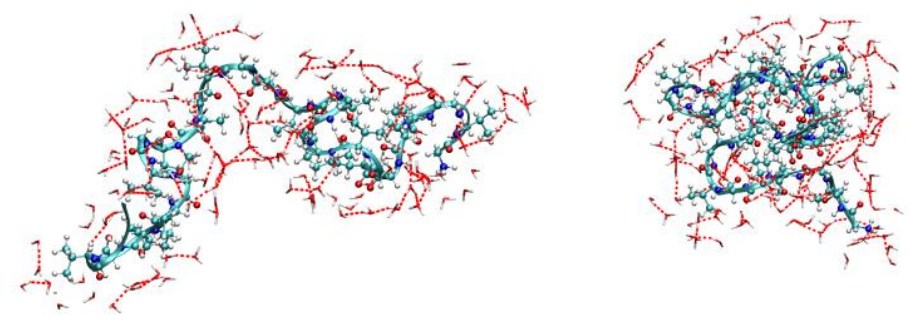

i)

j)

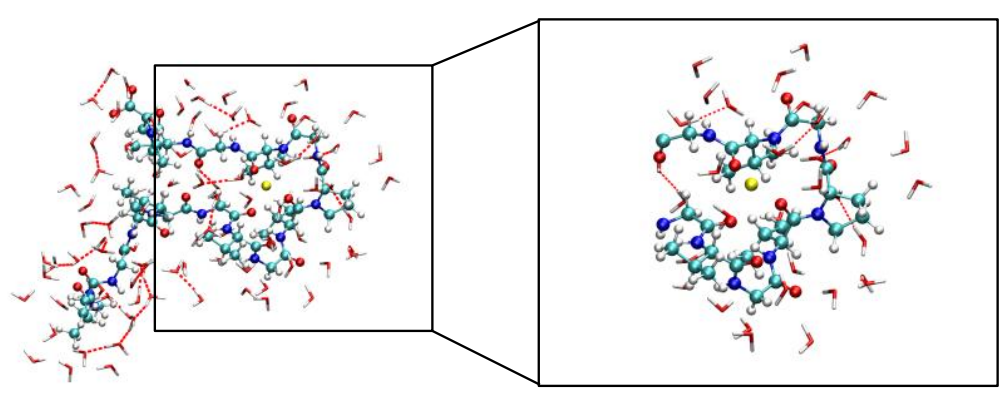

Figure 2 | Radius of gyration change with temperature. Green represents transition region; blue represents structures below transition range; red represents structures above transition range. a) (GVGVP) $)_{3}$ in $1 \mathrm{M} \mathrm{NaCl}$ with snapshots at low and high temperature; $b$ ) (GVGVP)(GKGVP)(GVGVP) in $1 \mathrm{M} \mathrm{NaCl}$; c) (GVGVP) 6 in $1 \mathrm{M} \mathrm{NaCl}$; d) (GVGVP) 3 in $100 \mathrm{mM}$ $\mathrm{NaCl}$; e) System 1 at $280 \mathrm{~K}$; f) System 1 at 340 K; g) System 3 at $280 \mathrm{~K}$; h) System 3 at 340 K; i) System 1 at $340 \mathrm{~K}$ showing peptide, hydrogen-bonded water molecules and a single sodium ion (in yellow) within a $3 \AA$ A solvation shell; j) Expanded image from (i) showing 
sodium ion (in yellow) interacting with electronegative atoms of the peptide. The sodium ion is hinged on the interaction with three oxygen atoms (in red). 

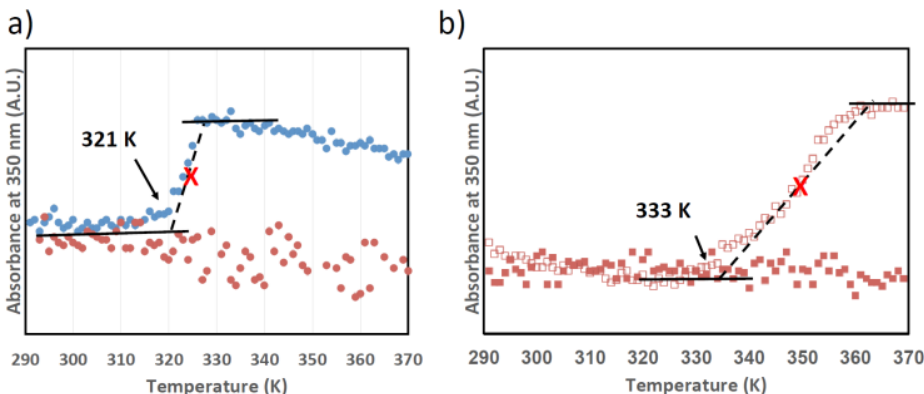

Figure 3 | (a) Turbidity profiles of (GVGVP)(GVGVP)(GVGVP) (blue dots) and (GVGVP)(GKGVP)(GVGVP) (orange dots) in deionized water, $40 \mathrm{mg} / \mathrm{ml}$, suggesting the transition temperature can be suppressed by switching $\mathrm{V}$ to $\mathrm{K}$, a charged residue. (b) Turbidity profiles of (GVGVP)(GKGVP)(GVGVP) in $0.1 \mathrm{M} \mathrm{NaCl}$ (orange squares) and $5 \mathrm{M} \mathrm{NaCl}$ (open orange squares), $40 \mathrm{mg} / \mathrm{ml}$, suggesting the transition temperature can be lowered by increasing the ionic strength. The black arrows represent the onsets of transition, and red crosses represent the inflection point. 
a)

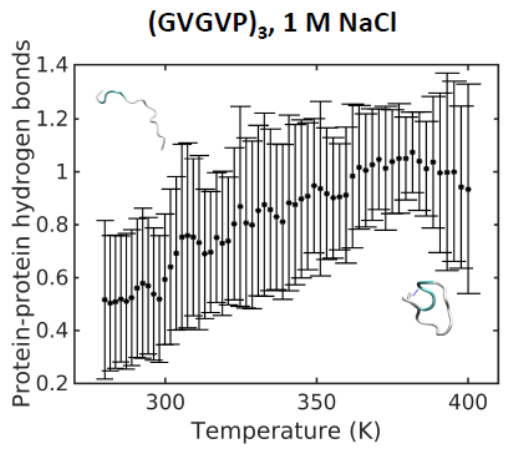

c)

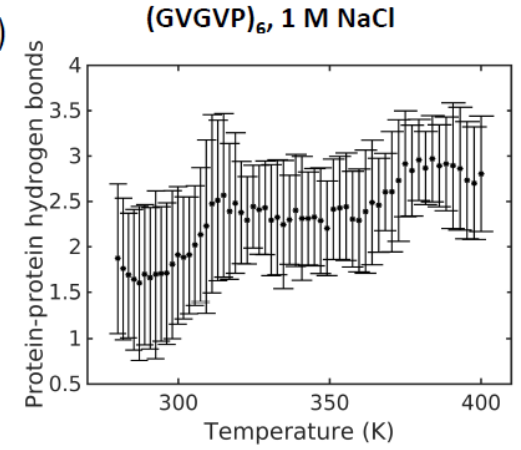

b)

(GVGVP)(GKGVP)(GVGVP), $1 \mathrm{M} \mathrm{NaCl}$

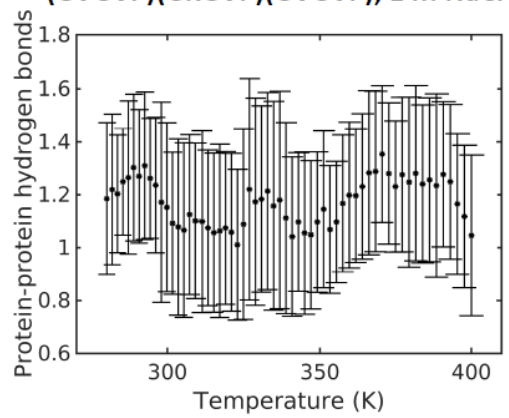

d)

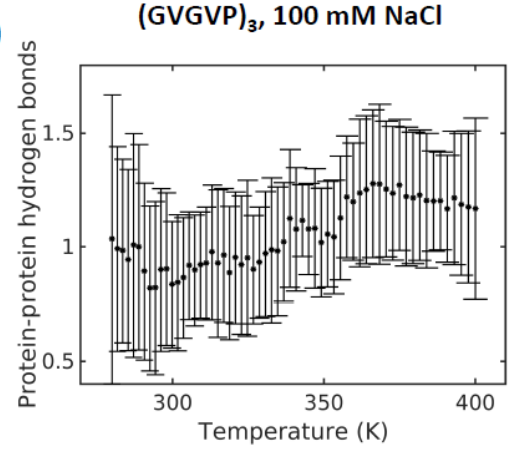

e)

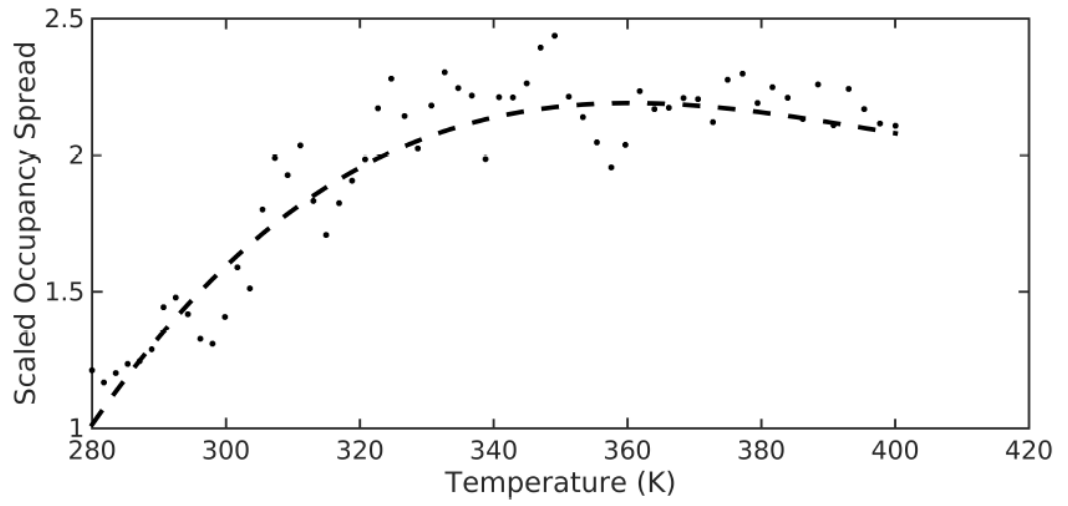

f)
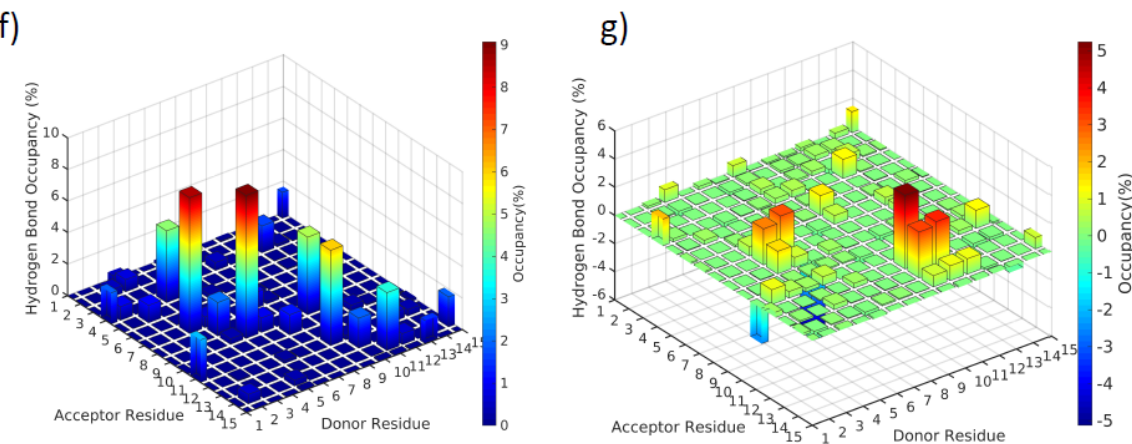

Figure 4 | Hydrogen bonds between protein residues change with temperature for a) $(G V G V P)_{3}$ in $1 \mathrm{M} \mathrm{NaCl}$ (cartoon representation insets show extended and folded states of the molecule), b) (GVGVP)(GKGVP)(GVGVP) in $1 \mathrm{M} \mathrm{NaCl}, \mathrm{c}$ ) (GVGVP) 6 in $1 \mathrm{M} \mathrm{NaCl}, \mathrm{d}$ ) (GVGVP) $)_{3}$ in $100 \mathrm{mM} \mathrm{NaCl}$; e) Scaled occupancy spread of hydrogen bonds between donor and acceptor residues as a function of temperature as defined by Equation 2; f) Hydrogen bond occupancy at $280 \mathrm{~K}$, defined as the fraction of time a hydrogen bond exists in the 
ensemble between a donor and an acceptor; g) Hydrogen bond occupancy difference between $340 \mathrm{~K}$ and $280 \mathrm{~K}$. 
a)

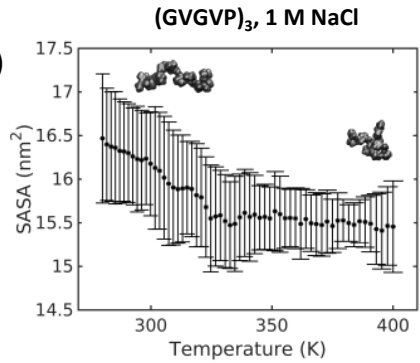

c)

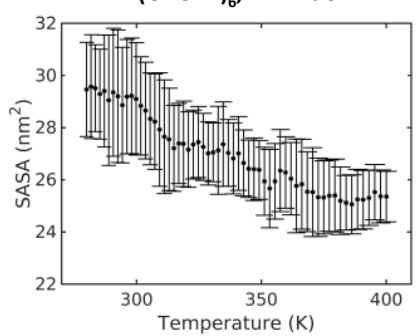

e)

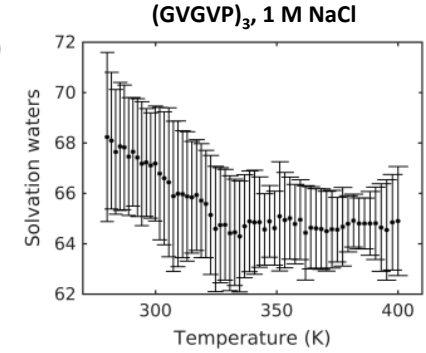

g)

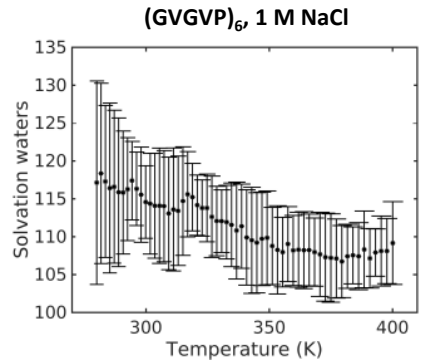

i)

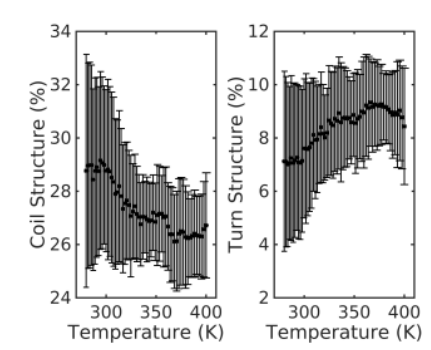

$($ GVGVP) $6,1 \mathrm{M} \mathrm{NaCl}$

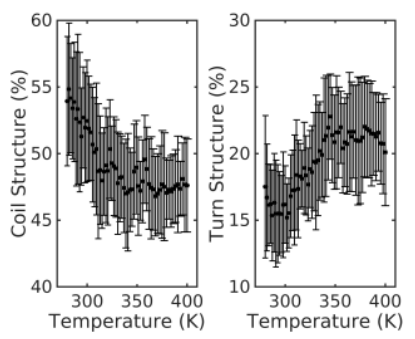

(GVGVP)(GKGVP)(GVGVP), $1 \mathrm{M} \mathrm{NaCl}$

b)

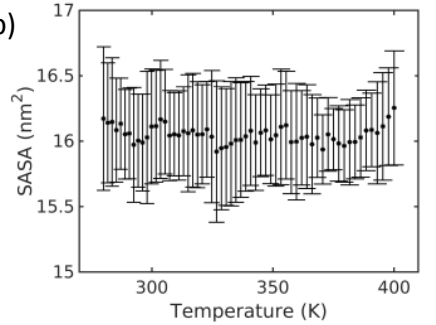

d)

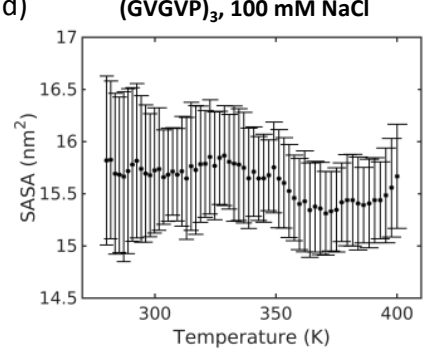

(GVGVP)(GKGVP)(GVGVP), $1 \mathrm{M} \mathrm{NaCl}$

f)

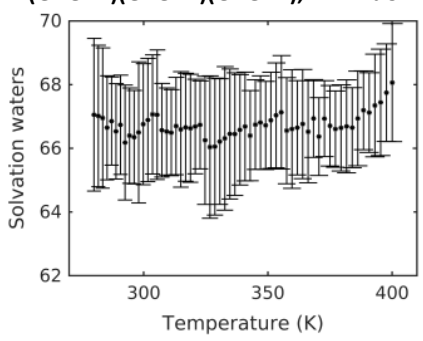

h)

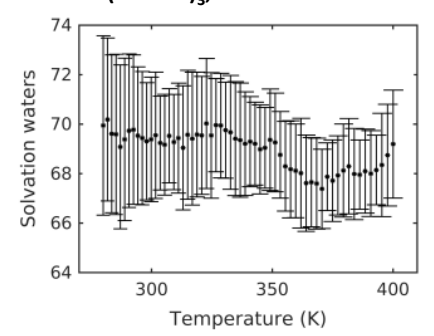

(GVGVP)(GKGVP)(GVGVP), $1 \mathrm{M} \mathrm{NaCl}$

j)

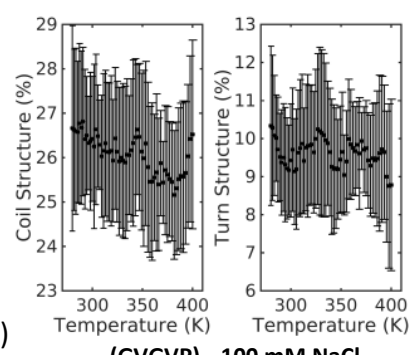

$(\text { GVGVP) })_{3}, 100 \mathrm{mM} \mathrm{NaCl}$
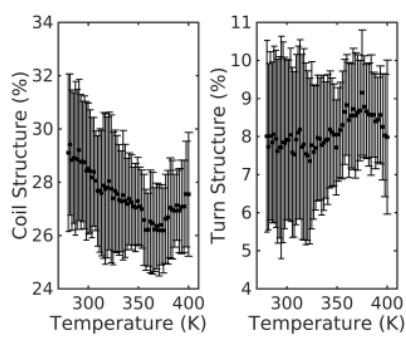
Figure 5 | Solvent accessible surface area (SASA) change with temperature for a) (GVGVP) 3 in $1 \mathrm{M} \mathrm{NaCl}$ with snapshots at low and high temperature (representation insets show SASA of extended and folded states of the molecule), b) (GVGVP)(GKGVP)(GVGVP) in $1 \mathrm{M} \mathrm{NaCl}, \mathrm{c}$ ) $(G V G V P)_{6}$ in $\left.1 \mathrm{M} \mathrm{NaCl}, \mathrm{d}\right)(\mathrm{GVGVP})_{3}$ in $100 \mathrm{mM} \mathrm{NaCl}$; e) Change with temperature of the number of water molecules within a $3 \AA$ solvation shell for (GVGVP) 3 in $1 \mathrm{M} \mathrm{NaCl,f}$ ) (GVGVP)(GKGVP)(GVGVP) in $1 \mathrm{M} \mathrm{NaCl}$, g) (GVGVP) 6 in $1 \mathrm{M} \mathrm{NaCl,} \mathrm{h}$ ) (GVGVP) $)_{3}$ in $100 \mathrm{mM}$ $\mathrm{NaCl}$; i) Coil and turn secondary structure changes with temperature for (GVGVP) 3 in $1 \mathrm{M}$ $\mathrm{NaCl}, \mathrm{j}$ ) (GVGVP)(GKGVP)(GVGVP) in $1 \mathrm{M} \mathrm{NaCl}, \mathrm{k}$ ) (GVGVP) 6 in $1 \mathrm{M} \mathrm{NaCl}, \mathrm{l}$ ) (GVGVP) 3 in 100 $\mathrm{mM} \mathrm{NaCl}$. 
a)

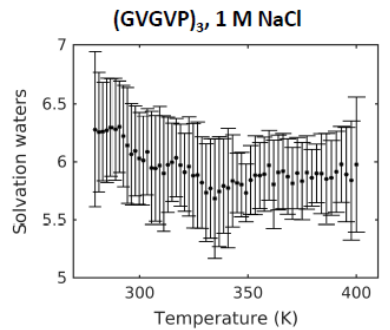

c)

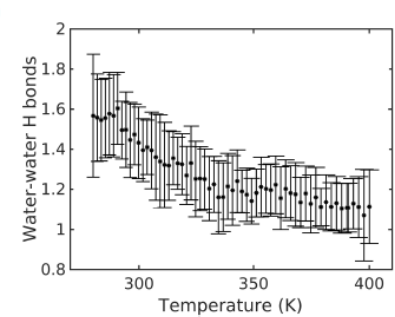

e)

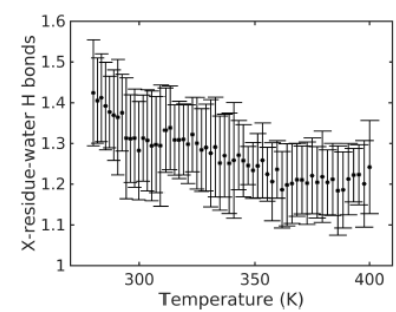

g)

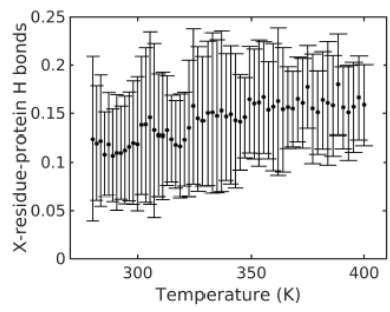

i)

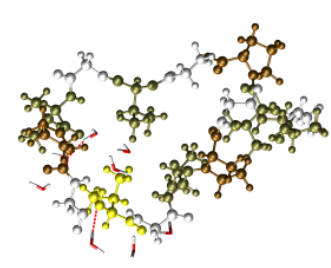

b)

(GVGVP)(GKGVP)(GVGVP), $1 \mathrm{M} \mathrm{NaCl}$

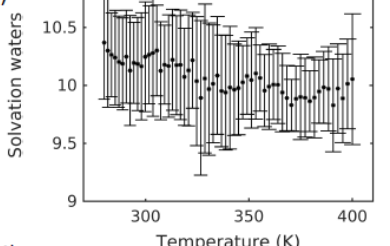

d)

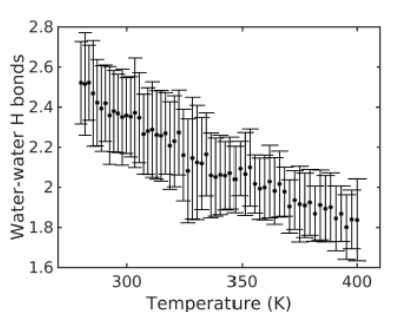

f)

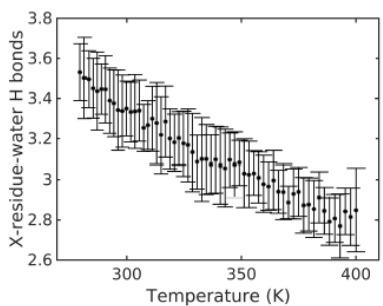

h)

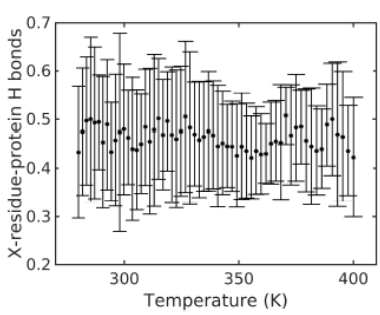

j)

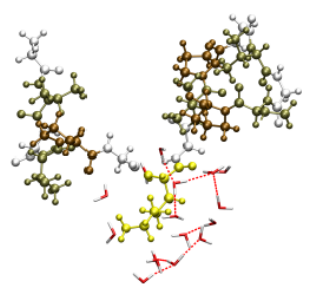

Figure 6 | a) Solvation waters within a $3 \AA$ A solvation shell of the seventh residue valine for System 1 and b) lysine for System 2. Water-water hydrogen bonds around the seventh residue of c) System 1 and d) System 2. Hydrogen bonds between water molecules and the seventh residue for e) System 1 and f) System 2. Hydrogen bonds formed between the peptide and the seventh residue of g) System 1 and h) System 2. Hydrogen bonded water molecules around the seventh residue (in yellow) of i) System 1 and j) System 2. 